\title{
CONGRUENCE REPRESENTATIONS OF JOIN-HOMOMORPHISMS OF FINITE DISTRIBUTIVE LATTICES: SIZE AND BREADTH
}

\author{
G. GRÄTZER, H. LAKSER and E. T. SCHMIDT
}

(Received 5 October 1998; revised 6 May 1999)

Communicated by B. A. Davey

\begin{abstract}
Let $K$ and $L$ be lattices, and let $\varphi$ be a homomorphism of $K$ into $L$. Then $\varphi$ induces a natural 0 -preserving join-homomorphism of Con $K$ into Con $L$.

Extending a result of Huhn, the authors proved that if $D$ and $E$ are finite distributive lattices and $\psi$ is a 0 -preserving join-homomorphism from $D$ into $E$, then $D$ and $E$ can be represented as the congruence lattices of the finite lattices $K$ and $L$, respectively, such that $\psi$ is the natural 0 -preserving join-homomorphism induced by a suitable homomorphism $\varphi: K \rightarrow L$. Let $m$ and $n$ denote the number of join-irreducible elements of $D$ and $E$, respectively, and let $k=\max (m, n)$. The lattice $L$ constructed was of size $O\left(2^{2(n+m)}\right)$ and of breadth $n+m$.

We prove that $K$ and $L$ can be constructed as 'small' lattices of size $O\left(k^{5}\right)$ and of breadth three.
\end{abstract}

1991 Mathematics subject classification (Amer. Math. Soc.): primary 06B10; secondary 06D05.

Keywords and phrases: Lattice, finite, congruence, distributive, breadth.

\section{Introduction}

The congruence lattice, Con $L$, of a finite lattice $L$ is a finite distributive lattice (Funayama and Nakayama [2]). The converse is a result of Dilworth, first published in Grätzer and Schmidt [9].

For a distributive lattice $D$ with $n$ join-irreducible elements, the original constructions (Dilworth's and also the one in Grätzer and Schmidt [9]) produced lattices of size $O\left(2^{2 n}\right)$ and of order dimension $O(2 n)$. In Grätzer and Lakser [4], this was improved

The research of the first and second authors was supported by the NSERC of Canada.

The research of the third author was supported by the Hungarian National Foundation for Scientific

Research, under Grant No. T023186.

(C) 2000 Australian Mathematical Society 0263-6115/2000\$A2.00+0.00 
to size $O\left(n^{3}\right)$ and order dimension 2 (therefore, planar and breadth 2). Finally, in Grätzer, Lakser, and Schmidt [5], a size $O\left(n^{2}\right)$ planar lattice was constructed.

THEOREM 1. Let $D$ be a finite distributive lattice with $n$ join-irreducible elements. Then there exists a planar lattice $L$ of $O\left(n^{2}\right)$ elements with $\operatorname{Con} L \cong D$.

Let $K$ and $L$ be lattices, and let $\varphi$ be a homomorphism of $K$ into $L$. Then $\varphi$ induces a map $\operatorname{Con} \varphi$ of $\operatorname{Con} K$ into $\operatorname{Con} L$ : for a congruence relation $\Theta$ of $K$, let the image of $\Theta$ under $\operatorname{Con} \varphi$ be the congruence relation of $L$ generated by the set $\Theta \varphi=\{\langle a \varphi, b \varphi\rangle \mid a \equiv b(\Theta)\}$.

The following result was proved by Huhn in [11] for embeddings and for arbitrary $\psi$ in Grätzer, Lakser, and Schmidt [7].

THEOREM 2. Let $D$ and $E$ be finite distributive lattices, and let

$$
\psi: D \rightarrow E
$$

be a 0 -preserving join-homomorphism. Then there are finite lattices $K$ and $L$, a lattice homomorphism $\varphi: K \rightarrow L$, and isomorphisms

$$
\alpha: E \rightarrow \operatorname{Con} L, \quad \beta:-D \rightarrow \operatorname{Con} K
$$

with

$$
\psi \alpha=\beta(\operatorname{Con} \varphi) .
$$

Furthermore, $\varphi$ is an embedding if and only if $\psi$ separates 0.

Theorem 2 concludes that the following diagram is commutative:

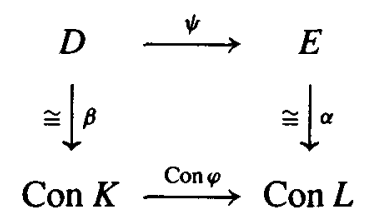

See Grätzer, Lakser, and Schmidt [6] for a short proof.

A lattice $L$ is said to be of breadth $p$, if $p$ is the smallest integer with the property that for every finite $X \subseteq L$, there exists a $Y \subseteq X$ such that $|Y| \leq p$ and $\wedge X=\wedge Y$. Note that this concept is self-dual. If $L$ is of breadth $p$, then for every finite $X \subseteq L$, there exists a $Y \subseteq X$ such that $|Y| \leq p$ and $\bigvee X=\bigvee Y$. If a finite lattice $L$ is of breadth $p$, then there is an element $a \in L$ with at least $p$ covers. The breadth of the Boolean lattice $C_{2}^{n}$ is $n$.

In this paper, we prove the following improvement of Theorem 2 along the lines of Theorem 1. 
THEOREM 3. Let $D$ be a finite distributive lattice with $n$ join-irreducible elements, let $E$ be a finite distributive lattice with $m$ join-irreducible elements, let $k=\max (m, n)$, and let

$$
\psi: D \rightarrow E
$$

be a 0-preserving join-homomorphism. Then there is a finite lattice $L$ of breadth 3 with $O\left(k^{5}\right)$ elements, a planar lattice $K$ with $O\left(n^{2}\right)$ elements, a lattice homomorphism $\varphi: K \rightarrow L$, and isomorphisms

$$
\alpha: E \rightarrow \operatorname{Con} L, \quad \beta: D \rightarrow \operatorname{Con} K
$$

with

$$
\psi \alpha=\beta(\operatorname{Con} \varphi)
$$

that is, such that the diagram

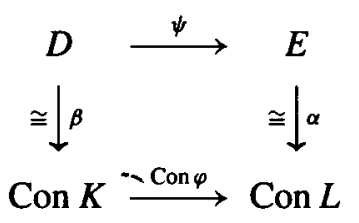

is commutative. Furthermore, $\varphi$ is an embedding if and only if $\psi$ separates 0.

In the last sentence of Theorem 3, ' $\psi$ separates 0 ' means that only the zero of $D$ is mapped under $\psi$ to the zero of $E$.

Outline Function lattices play a crucial role in the construction. Section 2 deals with function lattices, in general, while Section 3 discusses function lattices over $M_{3}$ and $N_{5}$. Actually, we need a somewhat more general construction, which we name generalized function lattices; these are discussed in Section 4.

Coloring is useful for the presentation of the first construction; it is introduced in Section 5.

The first construction produces the planar lattice $K$ of Theorem 3; it is borrowed from Grätzer, Lakser, and Schmidt [5] and briefly described in Section 6.

The second construction is based on multicoloring, introduced in Section 7; given a finite lattice $M$ and a multicoloring $\kappa$, we construct a generalized function lattice $M[\kappa]$.

The main construction is given, in four steps, in Section 8. The verification is presented in Section 9. Section 10 discusses Theorem 3 and the related open problems. 
Notation We use the notation of Grätzer [3].

$C_{n}$ denotes the $n$-element chain with $0<1<\cdots<n-1$. Let $N_{5}=\{o, a, b, c, i\}$, where $a<b$, denote the five-element nonmodular lattice and let $M_{3}=\{o, a, b, c, i\}$ be the five-element modular nondistributive lattice, both with zero $o$ and unit $i$.

\section{Function lattices, general observations}

For a lattice $M$, let $M^{C_{n}}$ denote the set of all order-preserving maps of $C_{n}$ to $M$, partially ordered by

$$
\alpha \leq \beta \quad \text { if an only if } x \alpha \leq x \beta \text {, for all } x \in C_{n} .
$$

Then $M^{C_{n}}$ is a lattice; it is called a function lattice. (In general, a function lattice $M^{P}$ is defined for any poset $P$.) The lattice $M^{C_{n}}$ is a subdirect product of $n$ copies of $M$; we shall use vector notation for the (isotone) maps. As illustrations, Figure 1 shows $N_{5}^{C_{3}}$ and Figure 2 depicts $M_{3}^{C_{2}}$.

In this section, we prove some general properties of function lattices.

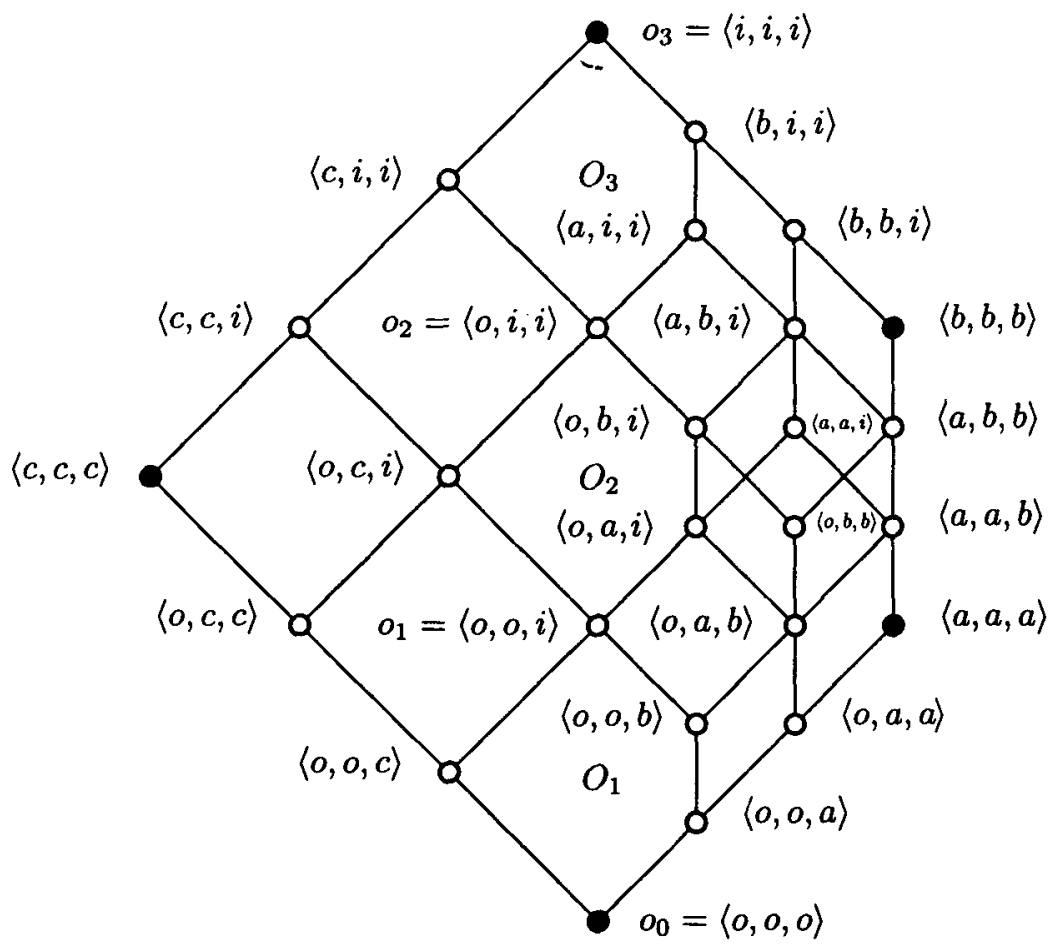

FIGURE 1. The lattice $N_{5}^{C_{3}}$. 


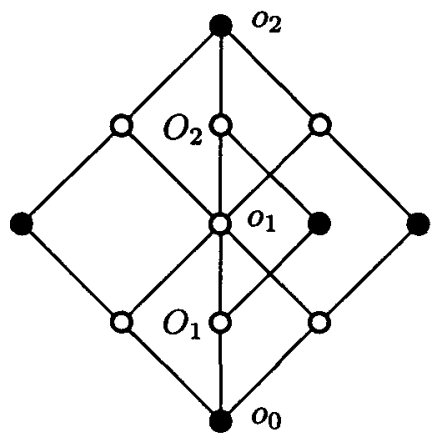

FIGURE 2. The lattice $M_{3}^{C_{2}}$.

LEMMA 1. $\left\langle a_{1}, \ldots, a_{n}\right\rangle \prec\left\langle b_{1}, \ldots, b_{n}\right\rangle$ in $M^{C_{n}}$ if an only if there exists a $k$ with $1 \leq k \leq n$ such that $a_{k} \prec b_{k}$ in $M$ and $a_{i}=b_{i}$, for $i \neq k$.

PROOF. Let $\left\langle a_{1}, \ldots, a_{n}\right\rangle \prec\left\langle b_{1}, \ldots, b_{n}\right\rangle$ in $M^{c_{n}}$. If there are $1 \leq k<l \leq n$ such that $a_{k}<b_{k}$ and $a_{l}<b_{l}$, then define $c_{i}=a_{i}$, for $i<l$ and $c_{i}=b_{i}$, for $i \geq l$. Obviously, $\left\langle a_{1}, \ldots, a_{n}\right\rangle<\left\langle c_{1}, \ldots, c_{n}\right\rangle$, since $a_{l}<b_{l}=c_{l}$, and $\left\langle c_{1}, \ldots, c_{n}\right\rangle<$ $\left\langle b_{1}, \ldots, b_{n}\right\rangle$, since $c_{k}=a_{k}<b_{k}$. The lemma now easily follows.

A sublattice of a finite lattice is called cover-preserving, if a prime interval of the sublattice is a prime interval of the whote lattice.

LEMMA 2. $M^{C_{n}}$ is a cover-preserving sublattice of $M^{n}$.

PROOF. Indeed, if $\left\langle a_{1}, \ldots, a_{n}\right\rangle \prec\left\langle b_{1}, \ldots, b_{n}\right\rangle$ in $M^{C_{n}}$, then by Lemma 1 , there exists a $k$ with $1 \leq k \leq n$ such that $a_{k} \prec b_{k}$ and $a_{i}=b_{i}$, for $i \neq k$. But then $\left\langle a_{1}, \ldots, a_{n}\right\rangle \prec\left\langle b_{1}, \ldots, b_{n}\right\rangle$ in $M^{n}$ is clear.

For $x \in M$, let $\mathbf{x}_{n}$ denote the constant function $\langle x, \ldots, x\rangle$ in $M^{C_{n}}$; if $n$ is clear from the context, it will be dropped. The constant maps form a sublattice of $M^{C_{n}}$; we identify $M$ with this sublattice. In Figure 1 and Figure 2, the elements of the form $\mathbf{x}$ are black filled.

LEMMA 3. If $\mathfrak{p}=[u, v]$ is a prime interval of $M$, then the corresponding interval $[\mathbf{u}, \mathbf{v}]$ of $M^{C_{n}}$ is isomorphic to $C_{n+1}$.

PROOF. The interval $[\mathbf{u}, \mathbf{v}]$ in $M^{C_{n}}$ consists of the elements

$$
\begin{aligned}
\mathbf{u}= & \langle u, u, \ldots, u, u\rangle,\langle u, u, \ldots, u, v\rangle,\langle u, u, \ldots, v, v\rangle, \ldots, \\
& \langle u, v, \ldots, v, v\rangle,\langle v, v, \ldots, v, v\rangle=\mathbf{v},
\end{aligned}
$$


and the coverings

$$
\begin{aligned}
\mathbf{u}= & \langle u, u, \ldots, u, u\rangle \prec\langle u, u, \ldots, u, v\rangle \prec\langle u, u, \ldots, v, v\rangle \prec \cdots \\
& \prec\langle u, v, \ldots, v, v\rangle \prec\langle v, v, \ldots, v, v\rangle=\mathbf{v}
\end{aligned}
$$

are clear from Lemma 1.

Take the following elements of $M^{n}$ :

$$
o_{i}=\langle 0, \ldots, 0, \underbrace{1, \ldots, 1}_{i}\rangle,
$$

for $0 \leq i \leq n$, where 0 and 1 is the zero and unit of $M$, respectively. Then $M$ is naturally isomorphic to the interval $O_{i}=\left[o_{i-1}, o_{i}\right] \subseteq M^{n}$, for $1 \leq i \leq n$, under the isomorphism

$$
x \longrightarrow\langle 0, \ldots, 0, x, \underbrace{1, \ldots, 1}_{i}\rangle, \quad x \in M .
$$

Observe that all these elements belong to $M C^{C_{3}}$, hence the intervals $O_{i}=\left[o_{i-1}, o_{i}\right] \subseteq$ $M^{C_{n}}$, for $1 \leq i \leq n$. (These elements and intervals are marked in Figure 1 and Figure 2.) So, we can consider $M^{C_{n}}$ as a subdirect product of the $O_{i}, 1 \leq i \leq n$, that is, a sublattice of $\prod\left(O_{i} \mid 1 \leq i \leq n\right) \cong M^{n}$. Let $O(M)$ denote the sublattice $\bigcup\left(O_{i} \mid i \leq n\right)$ of $M^{n}$; note that $O(M)$ is a sublattice of $M^{C_{n}}$.

A finite lattice $K$ is a congruence-preserving extension of $L$, if $L$ is a sublattice of $K$ and every congruence of $L$ has exactly one extension to $K$. Of course, then the congruence lattice of $L$ is isomorphic to the congruence lattice of $K$.

LEMMA 4. Let $E$ be a sublattice of $M^{n}$ containing $O(M)$. Then $M^{n}$ is a congruence-preserving extension of $E$. In particular, $M^{n}$ is a congruence-preserving extension of $M^{C_{n}}$.

PROOF. Let $\Theta$ be a congruence relation of $E$. Since $O_{i} \subseteq E$, for $1 \leq i \leq n$, we can restrict $\Theta$ to $O_{i}$, to obtain the congruence $\Theta_{i}$. Then $\prod\left(\Theta_{i} \mid 1 \leq i \leq n\right)$ is (up to isomorphism) a congruence of $M^{n}$ that extends $\Theta$. To show the uniqueness of the extension, let $\Phi$ be a congruence of $M^{n}$ that extends $\Theta$. Then $\Phi$ restricted to any $O_{i}$ will agree with $\Theta$ restricted to $O_{i}$, hence $\Phi=\Theta$.

Observe that this proof holds for function lattices (with finite exponents, $P$ ), in general, so we obtain a result of Duffus, Jónsson, and Rival [1]: $\operatorname{Con} M^{P} \cong(\operatorname{Con} M)^{n}$, where $n=|P|$. 


\section{Function lattices over $M_{3}$ and $N_{5}$}

In this section, we investigate, in detail, the cases $M=M_{3}$ and $M=N_{5}$. See Figure 1 and Figure 2, for illustration. Note that $N_{5}^{C_{2}}$ is planar, that is why we show $N_{5} C_{3}$.

The structure of $M_{3}^{C_{n}}$ is rather well known (Schmidt [12]).

LEMMA 5. $M_{3}^{c_{n}}$ is a modular lattice containing $\{\mathbf{o}, \mathbf{a}, \mathbf{b}, \mathbf{c}, \mathbf{i}\}$ as a $\{0,1\}$-sublattice isomorphic to $M_{3}$. The interval $[\mathbf{0}, \mathbf{a}]$ is isomorphic to $C_{n+1}$ and $M_{3}^{C_{n}}$ is a congruencepreserving extension of the chain $[\mathbf{0}, \mathbf{a}] \cong C_{n+1}$. In particular, every prime interval of $M_{3}^{C_{n}}$ is projective to a prime interval of $[\mathbf{0}, \mathbf{a}]$.

Now we proceed to describe the structure of $N_{5}^{C_{n}}$.

LEMMA 6. Let $\Theta$ be the kernel of the $n$-th projection on $N_{5}^{C_{n}}$, that is, of the homomorphism $\left\langle x_{1}, \ldots, x_{n}\right\rangle \rightarrow x_{n}$ of $N_{5}^{C_{n}}$ to $N_{5}$. Let $A_{x}, x \in\{o, a, b, c, i\}$, denote the five congruence classes $\left(A_{x}\right.$ contains $\left.\mathbf{x}\right)$. Then

(i) $A_{o}=\{\mathbf{0}\}$

(ii) $A_{a}=\left[\langle o, \ldots, o, a\rangle\right.$, a] $\cong C_{n}$;

(iii) $A_{b}=\left[\langle o, \ldots, o, b\rangle\right.$, b] $\cong C_{3}^{C_{n-1}}$;

(iv) $A_{c}=[\langle o, \ldots, o, c\rangle, \mathrm{c}] \cong C_{n}$;

(v) $A_{i}=[\langle o, \ldots, o, i\rangle, \mathbf{i}] \cong N_{5}^{C_{n-1}} ;$

(vi) $A_{a}$ is isomorphic to $\left[\mathbf{o}_{n-1}, \mathbf{a}_{n-1}\right] \subseteq N_{5}^{C_{n-1}}$;

(vii) $A_{b}$ is isomorphic to $\left[\mathbf{o}_{n-1}, \mathbf{b}_{n-1}\right] \subseteq N_{5}^{C_{n-1}}$;

(viii) $A_{c}$ is isomorphic to $\left[\mathbf{0}_{n-1}, \mathbf{c}_{n-1}\right] \subseteq N_{5}^{C_{n-1}}$;

(ix) $A_{o} \cup A_{a} \cup A_{b} \cong C_{3}^{C_{n}}$.

Moreover,

(x) $A_{a} \cup[\langle o, \ldots, o, b\rangle,\langle a, \ldots, a, b\rangle] \cup[\langle o, \ldots, o, i\rangle,\langle a, \ldots, a, i\rangle]$ $\cong A_{a} \times C_{3} \cong\left[\mathbf{o}_{n-1}, \mathbf{a}_{n-1}\right] \times C_{3}$

(xi) $A_{b} \cup[\langle o, \ldots, o, i\rangle,\langle b, \ldots, b, i\rangle] \cong A_{b} \times C_{2} \cong\left[\mathbf{o}_{n-1}, \mathbf{b}_{n-1}\right] \times C_{2}$;

(xii) $A_{c} \cup[\langle o, \ldots, o, i\rangle,\langle c, \ldots, c, i\rangle] \cong A_{c} \times C_{2} \cong\left[\mathbf{o}_{n-1}, \mathbf{c}_{n-1}\right] \times C_{2}$.

PROOF. Obvious, by direct computation.

For a finite lattice $M$, an edge $E_{\mathfrak{p}}$ of $M^{C_{n}}$ is an interval $[\mathbf{u}, \mathbf{v}]$ of $M^{C_{n}}$, where $\mathfrak{p}=[u, v]$ is a prime interval of $M$.

LEMMA 7. Every prime interval $\mathfrak{p}$ of $N_{5}^{C_{n}}$ is projective to a prime interval $\mathfrak{q}$ in one of the edges [o, a], [a, b], [b, i] of $N_{5}^{C_{n}}$.

PROOF. We prove this by induction on $n$. 
If $n=1$, then every prime interval is either one of the edges listed or it is projective to one of the edges listed by Lemma 6 .

Let us assume that the statement is proved for $n-1$. Let $\mathfrak{p}$ be a prime interval of $N_{5}^{C_{n}}$. We partition $N_{5}^{C_{n}}$ as in Lemma 6 into the sets $A_{x}, x \in\{o, a, b, c, i\}$. Then $A_{i} \cong N_{5}^{C_{n-1}}$, so the statement of this lemma is assumed for $A_{i}$.

Let $\mathfrak{p}=[u, v]$ be a prime interval of $N_{5}^{C_{n}}$.

First, let $\mathfrak{p} \subseteq A_{i}$. For a prime interval $\mathfrak{q}$ of $N_{5}$, let $E_{\mathfrak{q}, i-1}$ and $E_{\mathfrak{q}, i}$ be the corresponding edges of $A_{i}$ and $N_{5}^{C_{n}}$, respectively. By Lemma 6 , either $E_{\mathrm{q}, i-1} \subseteq E_{\mathrm{q}, i}$, or $E_{\mathbf{q}, i-1}$ and $E_{\mathbf{q}, i}$ are contained in a distributive sublattice of $N_{5}^{C_{n}}$, in which every prime interval of $E_{\mathfrak{q}, i-1}$ is perspective to a prime interval of $E_{\mathfrak{q}, i}$; so the statement follows for $\mathfrak{p}$.

Second, let $\mathfrak{p} \nsubseteq A_{i}$. If $\mathfrak{p} \subseteq A_{o} \cup A_{c}$, then the statement is trivial since the prime interval is perspective to one in [b, i].

If $\mathfrak{p} \subseteq A_{o} \cup A_{a} \cup A_{b}$, then the statement is trivial since the edges of $N_{5}^{C_{n}}$ in this distributive lattice form maximal chains.

Finally, if $u \in A_{c}$ or $u \in A_{b}$ and $v \in A_{i}$, then pick $\mathfrak{q}=\left[\mathbf{o}_{n}, w\right]$, where $w$ is the least element of $A_{a}$ or of $A_{c}$, respectively, and observe that $\mathfrak{q}$ is in the edge $[\mathbf{0}, \mathbf{a}]$ or it is perspective to a prime interval in the edge $[\mathbf{b}, \mathbf{i}]$.

Finally, in this section, we look at size and Breadth.

LEMMA 8. $N_{5}^{C_{n}}$ and $M_{3}^{C_{n}}$ are lattices of breadth 3. The lattice $N_{5}^{C_{n}}$ has $O\left(n^{3}\right)$ elements and $M_{3}^{C_{n}}$ has $O\left(n^{2}\right)$ elements.

PROOF. An arbitrary element of $N_{5}^{C_{n}}$ has either the form

$$
\langle\underbrace{o, \ldots, o}_{i}, \underbrace{a, \ldots, a}_{j}, \underbrace{b, \ldots, b}_{k}, \underbrace{i, \ldots, i}_{t}\rangle
$$

where $i+j+k+l=n(0 \leq i, j, k, l \leq n)$ or the form

$$
\langle\underbrace{o, \ldots, o}_{i}, \underbrace{c, \ldots, c}_{j}, \underbrace{i, \ldots, i}_{k}\rangle
$$

where $i+j+k=n(0 \leq i, j, k \leq n)$.

To prove the first statement of the lemma, we prove the stronger statement that an element of $N_{5}^{C_{n}}$ can have at most three upper covers. We get an upper cover of $u$, represented as in (1), by replacing the last $o$ by $a$, or the last $a$ by $b$, or the last $b$ by $i$, proving the statement for $u$. The proof for an element $u$ represented as in (2) is similar.

The number of elements of $N_{5}^{C_{n}}$ represented as in (1) is the number of ways we can choose $i, j$, and $k$ so that $i+j+k+l=n$, for some $l$; there are $O\left(n^{3}\right)$ choices. 
Similarly, the number of elements of $N_{5}^{C_{n}}$ represented as in (2) is $O\left(n^{2}\right)$, proving both statements for $N_{5}^{C_{n}}$. The proof for $M_{3}^{C_{n}}$ is similar.

\section{Generalized function lattices}

For a lattice $M$, a finite chain $C_{n}$, and congruences $\Theta_{1}, \ldots, \Theta_{n}$ of $M$, a generalized function lattice over $M$ is the sublattice of $M / \Theta_{1} \times \cdots \times M / \Theta_{n}$ defined by

$$
\left\{\left\langle\left[a_{1}\right] \Theta_{1}, \ldots,\left[a_{n}\right] \Theta_{n}\right\rangle \mid\left\langle a_{1}, \ldots, a_{n}\right\rangle \in M^{C_{n}}\right\} .
$$

Equivalently, let $\Theta$ be a congruence of $M^{C_{n}}$; by Lemma 4, $\Theta$ can be described by the restrictions $\Theta_{1}, \ldots, \Theta_{n}$ of $\Theta$ to the intervals $O_{1}, \ldots, O_{n}$. The generalized function lattice defined in the previous paragraph is isomorphic to $M^{C_{n}} / \Theta$.

Now we borrow the arguments of Lemma 1 and Lemma 2.

LEMMA 9. The covering relation

$$
\left\langle\left[a_{1}\right] \Theta_{1}, \ldots,\left[a_{n}\right] \Theta_{n}\right\rangle \prec\left\langle\left[b_{1}\right] \Theta_{1}, \ldots,\left[b_{n}\right] \Theta_{n}\right\rangle
$$

holds in the generalized function lattice if and only if there exists a $k$ with $1 \leq k \leq n$ such that $\left[a_{k}\right] \Theta_{k} \prec\left[b_{k}\right] \Theta_{k}$ in $M / \Theta_{k}$ and $\left[a_{i}\right] \Theta_{i}=\left[b_{i}\right] \Theta_{i}$, for $i \neq k$.

LEMMA 10. The generalized function lattice is a cover-preserving sublattice of $M / \Theta_{1} \times \cdots \times M / \Theta_{n}$.

To prove these two lemmas, observe that if

$$
\left\langle\left[a_{1}\right] \Theta_{1}, \ldots,\left[a_{n}\right] \Theta_{n}\right\rangle \leq\left\langle\left[b_{1}\right] \Theta_{1}, \ldots,\left[b_{n}\right] \Theta_{n}\right\rangle,
$$

then

$$
\left\langle\left[b_{1}\right] \Theta_{1}, \ldots,\left[b_{n}\right] \Theta_{n}\right\rangle=\left\langle\left[a_{1} \vee b_{1}\right] \Theta_{1}, \ldots,\left[a_{n} \vee b_{n}\right] \Theta_{n}\right\rangle,
$$

and $\left\langle a_{1} \vee b_{1}, \ldots, a_{n} \vee b_{n}\right\rangle \in M^{C_{n}}$, so we can assume without the loss of generality that $a_{i} \leq b_{i}, 1 \leq i \leq n$. Now we can follow the arguments of Lemma 1 and Lemma 2 , mutatis mutandis.

Similarly, we can borrow the argument of Lemma 8:

LEMMA 11. A generalized function lattice over $N_{5}$ is of breadth 3.

We do not need the corresponding statement for $M_{3}$ since every generalized function lattice over $M_{3}$ is a function lattice over $M_{3}$. 


\section{Coloring}

Let $M$ be a finite lattice, and let $Q$ be a finite set; the elements of $Q$ will be called colors. Following Teo [13], a coloring $\mu$ of $M$ over $Q$ is a map

$$
\mu: \mathfrak{P}(M) \rightarrow Q
$$

of the set of prime intervals $\mathfrak{P}(M)$ of $M$ into $Q$ satisfying the condition: if two prime intervals generate the same congruence relation of $M$, then they have the same color; that is,

$$
\mathfrak{p}, \mathfrak{q} \in \mathfrak{P}(M) \text { and } \Theta(\mathfrak{p})=\Theta(\mathfrak{q}) \text { imply that } \mathfrak{p} \mu=\mathfrak{q} \mu .
$$

Since the join-irreducible congruences of $M$ are exactly those that can be generated by prime intervals, equivalently, $\mu$ can be regarded as a map of the set $\mathrm{J}(\operatorname{Con} M)$ of join-irreducible congruences of $M$ into $Q$ :

$$
\mu: \mathrm{J}(\operatorname{Con} M) \rightarrow Q .
$$

If all prime intervals of $M$ have the same color $q \in Q$, then we speak of a monochromatic lattice of color $q$.

We shall define a coloring by specifying $\mu$ on a large enough subset of $\mathfrak{P}(M)$ so that for every prime interval of $M$ there is one in the subset that generates the same congruence.

Let $M_{i}$ be a lattice colored by $\mu_{i}$ over $Q_{i}$, for $1 \leq i \leq n$. Then $\prod\left(M_{i} \mid 1 \leq i \leq n\right)$ has a natural coloring over $\bigcup\left(Q_{i} \mid 1 \leq i \leq n\right)$, since every prime interval of $\prod\left(M_{i} \mid 1 \leq i \leq n\right)$ is uniquely associated with a $k, 1 \leq k \leq n$, and a prime interval of $M_{k}$.

DEFINITION 1. We call $M \subseteq \prod\left(M_{i} \mid 1 \leq i \leq n\right)$ a colored subdirect product of the $M_{i}, 1 \leq i \leq n$, if the following conditions are satisfied:

(i) $M$ is a subdirect product of the $M_{i}, 1 \leq i \leq n$;

(ii) $M$ is a cover-preserving sublattice of $\prod\left(M_{i} \mid 1 \leq i \leq n\right)$;

(iii) the coloring of $M$ is the coloring inherited from the coloring of $\prod\left(M_{i} \mid 1 \leq\right.$ $i \leq n)$.

By Lemma 2, if $M$ is colored over $Q$, then $M^{C_{n}}$ is also colored over $Q$.

\section{The first construction: a planar lattice}

The proof of Theorem 3 starts with the planar construction of Grätzer, Lakser, and Schmidt [5]. We shall outline it in a somewhat simplified form. 
Let $D$ be a finite distributive lattice, and let $J=\mathrm{J}(D)=\left\{d_{1}, \ldots, d_{n}\right\}$ be the set of join-irreducible elements of $D$. Let $S_{0}$ be a chain of length $2 n$. We color the prime intervals of $S_{0}$ over $J$ as follows: we color the lower-most two prime intervals of $S_{0}$ with $d_{1}$, the next two with $d_{2}$, and so on. For each $d \in J$, there is a unique subchain $d^{b} \prec d^{m} \prec d^{t}$ of $S_{0}$ such that the prime intervals $\left[d^{b}, d^{m}\right]$ and $\left[d^{m}, d^{t}\right]$ have color $d$, and no other prime interval of $S_{0}$ has color $d$.

Let $S_{1}$ be a chain of length $n$. We color the prime intervals of $S_{1}$ by an arbitrary bijection. Thus, for each $d \in J$, there is in $S_{1}$ exactly one prime interval of color $d$; we denote it by $\left[d^{o}, d^{i}\right]$.

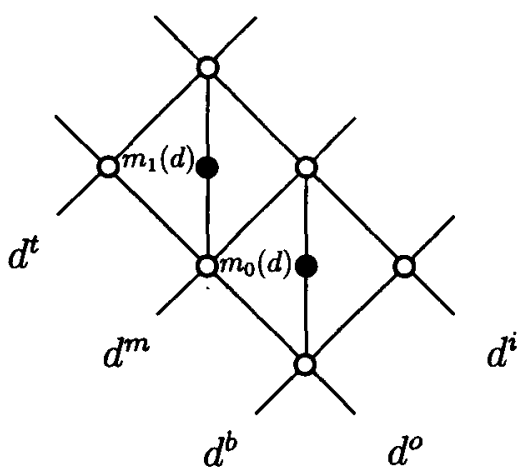

FIGURE 3. Adding the elements $m_{0}(d)$ and $m_{1}(d)$.

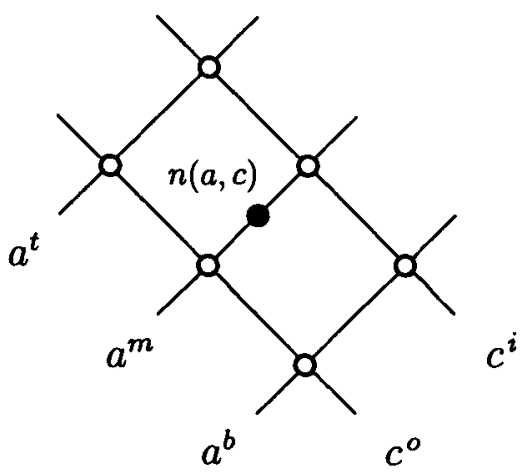

FIGURE 4. Adding the element $n(a, c)$.

We set $K_{0}=S_{0} \times S_{1}$. We shall regard $S_{0}$ and $S_{1}$ as sublattices of $K_{0}$, in the usual manner. We extend the lattice $K_{0}$ to a lattice $K$ : for each $d \in J$, we adjoin two new elements $m_{0}(d)$ and $m_{1}(d)$, as illustrated in Figure 3, and for each pair $a>c$ in $J$, we add a new element $n(a, c)$, as illustrated in Figure 4. To $d \in J$, assign the congruence of $K$ generated by any/all prime intervals of this color. This defines an isomorphism 

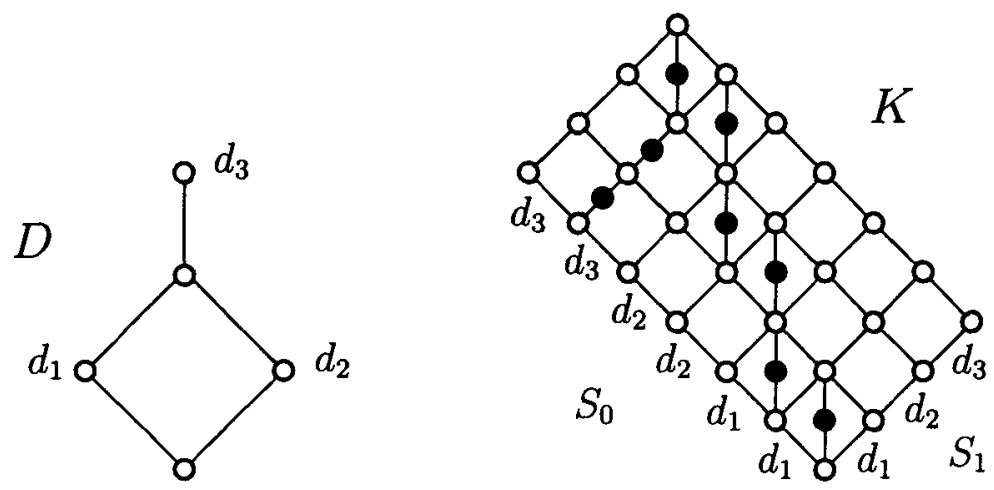

FIGURE 5. The lattice $X$ constructed from $D$.

between $J$ and the poset of join-irreducible congruences of $K$; consequently, the congruence lattice of $L$ is isomorphic to $D$.

Note that $K$ is a planar lattice and $|K|<3(n+1)^{2}$.

For instance, if $D$ is the five-element distributive lattice of Figure 5 , then $\mathrm{J}(D)$ is the poset $\left\{d_{1}, d_{2}, d_{3}\right\}$ with $d_{1}<d_{3}, d_{2}<d_{3}$, and we obtain the lattice $K$ of Figure 5.

\section{Multicoloring and the second construction}

Let $M$ be a finite lattice, and let $Q$ be a finite set. A multicoloring of $M$ over $Q$ is an isotone map $\mu$ from $\mathfrak{P}(M)$ into $P^{+}(Q)$ (the set of all nonempty subsets of $Q$ ); isotone means that if $\mathfrak{p}, \mathfrak{q} \in \mathfrak{P}(M)$ and $\Theta(\mathfrak{p}) \leq \Theta(\mathfrak{q})$, then $\mathfrak{p} \mu \subseteq \mathfrak{q} \mu$. Equivalently, a multicoloring is an isotone map of the poset $\mathrm{J}(\operatorname{Con} M)$ into the poset $P^{+}(Q)$.

The second construction starts with a lattice $M$ multicolored by $\kappa$ and constructs a generalized function lattice $M[\kappa]$ with coloring $\mu[\kappa]$. The lattice $M$ embeds into $M[\kappa]$ such that the congruence structure of $M[\kappa]$ is easy to work with and $\kappa$ is determined by $\mu[\kappa]$. We construct $M[\kappa]$ as a generalized function lattice.

Let $M$ be a finite lattice with a multicoloring $\kappa$ over the $n$-element set $Q=$ $\left\{q_{1}, q_{2}, \ldots, q_{n}\right\}$. For any $k$ with $1 \leq k \leq n$, define the binary relation $\Phi_{k}$ on $M$ as follows:

$u \equiv v\left(\Phi_{k}\right)$ if and only if $q_{k} \notin \mathfrak{p} \kappa$, for any prime interval $\mathfrak{p} \subseteq[u \wedge v, u \vee v]$.

LEMMA 12. $\Phi_{k}$ is a congruence relation on $M$.

PROOF. The relation $\Phi_{k}$ is obviously reflexive and symmetric. To show the transitivity of $\Phi_{k}$, assume that $u \equiv v\left(\Phi_{k}\right)$ and $v \equiv w\left(\Phi_{k}\right)$, and let $q$ be a prime interval in $[u \wedge w, u \vee w]$. Then $\mathfrak{q}$ is collapsed by $\Theta(u, v) \vee \Theta(v, w)$, hence there is a prime 
interval $\mathfrak{p}$ in $[u \wedge v, u \vee v]$ or in $[v \wedge w, v \vee w]$ satisfying $\Theta(\mathfrak{q}) \leq \Theta(\mathfrak{p})$. It follows from the definition of multicoloring that $\mathfrak{q} \kappa \subseteq \mathfrak{p} \kappa$; since $q_{k} \notin \mathfrak{p} \kappa$, it follows that $q_{k} \notin \mathfrak{q} \kappa$, hence $u \equiv w\left(\Phi_{k}\right)$. The proof of the Substitution Property is similar.

We define $M[\kappa]$ as the generalized function lattice over $M$ determined by the congruences $\Phi_{1}, \ldots, \Phi_{n}$. Set $M_{i}=M / \Phi_{i}$, for $1 \leq i \leq n$.

For $a \in M$, define

$$
a[\kappa]=\left\langle[a] \Phi_{1}, \ldots,[a] \Phi_{n}\right\rangle .
$$

Then the map $a \rightarrow a[\kappa]$ maps $M$ into $M[\kappa]$.

For $1 \leq i \leq n$, we color the lattice $M_{i}$ (monochromatically) by $q_{i}$. So we can regard $M_{1} \times \cdots \times M_{n}$ as colored over $Q$. By Lemma $10, M[\kappa]$ is a cover-preserving sublattice of $M_{1} \times \cdots \times M_{n}$, so $M[\kappa]$ inherits the coloring, which we shall denote by $\mu[\kappa]$.

Let us color the chain $C_{n+1}$ by $Q$ as follows: the color of the prime interval $[i-1, i]$ of $C_{n+1}$ is $q_{i}$, for $1 \leq i \leq n$. For a prime interval $\mathfrak{p}=[a, b]$ in $M$, we denote by $C_{n+1, p}$ the homomorphic image of $C_{n+1}$ obtained by collapsing all prime intervals of color not in $\mathrm{p} \kappa$.

The following lemma states the most important properties of $M[\kappa]$.

LEMMA 13. $M[\kappa]$ with the coloring $\mu[\kappa]$ over $Q$ has the following properties:

(i) $M[\kappa]$ with the coloring $\mu[\kappa]$ is a colored subdirect product of the monochromatic lattices $M_{i}$, of color $q_{i}, 1 \leq i \leq n$.

(ii) The map $a \rightarrow a[\kappa]$ is a lattice embedding of $M$ into $M[\kappa]$.

(iii) For any prime interval $\mathfrak{p}=[a, b]$ in $M$, the interval $[a[\kappa], b[\kappa]]$ is isomorphic to $C_{n+1, p}$.

(iv) The coloring $\mu[\kappa]$ of $M[\kappa]$ determines the multicoloring $\mu$ of $M$, namely, for every prime interval $\mathfrak{p}=[a, b]$ of $M$,

$$
\mathfrak{p} \mu=\{\mathfrak{q} \mu[\kappa] \mid \mathfrak{q} \in \mathfrak{P}(M[\kappa]) \text { and } \mathfrak{q} \subseteq[a[\kappa], b[\kappa]]\} .
$$

(v) For any prime interval $\mathfrak{p}=[a, b]$ in $M[\kappa]$, there is a unique $k$ with $1 \leq k \leq n$, and a prime interval $\mathfrak{q}$ in $M_{k}$ such that $\mathfrak{p}$ is projective to $\mathfrak{q}$. Define a congruence relation

$$
\Phi_{\mathfrak{p}}=\omega_{1} \times \cdots \times \omega_{k-1} \times \Theta(\mathfrak{q}) \times \omega_{k+1} \times \cdots \times \omega_{n}
$$

of $\prod\left(M_{i} \mid 1 \leq i \leq n\right)$, where $\omega_{j}$ is the trivial congruence $\omega$ on $M_{j}$, for $j \neq k$. Then $\Theta(p)$ is the restriction of $\Phi_{\mathfrak{p}}$ to $M[\kappa]$.

(vi) The congruence lattice of $M[\kappa]$ is described by the following formula:

$$
\operatorname{Con} M[\kappa] \cong \prod\left(\operatorname{Con} M_{i} \mid 1 \leq i \leq n\right) .
$$


PROOF. (i) and (iii) obviously hold.

(ii) The map $a \rightarrow a[\kappa]$ is a lattice homomorphism. We have to prove that it is one-to-one. Let $a, b \in M$ and $a \neq b$; we have to prove that $a[\kappa] \neq b[\kappa]$. Let $\mathfrak{p}$ be a prime interval in $[a \wedge b, a \vee b]$. Since $\mu[\kappa]$ is a multicoloring, there is a $q_{i} \in \mathfrak{p} \mu[\kappa]$. Obviously, then $a \not \equiv b\left(\bmod \Phi_{i}\right)$, from which the statement follows.

(iv) Let $a \prec b$ in $M$. Then [a, b] in $M^{C_{n}}$ is isomorphic to $C_{n+1}\left(\cong C_{2}^{C_{n}}\right)$. By the definition of $\Phi_{i}, 1 \leq i \leq n$, we get the fourth statement.

(v) and (vi) are also trivial.

Let $A=[a, b]$ be an interval of $M$. Then the multicoloring $\kappa$ of $M$ defines a multicoloring $\kappa_{A}$ on $\mathrm{A}$; so the lattice $A\left[\kappa_{A}\right]$ is defined. On the other hand, $A$ is a sublattice of $M[\kappa]$ (by identifying $x \in A$ with $x[\kappa]$ ), so it defines an interval $(A)_{M[\kappa]}=[a[\kappa], b[\kappa]]$ of $M[\kappa]$.

LEMMA 14. The lattices, $A\left[\kappa_{A}\right]$ and $(A)_{M[\kappa]}$ are isomorphic.

ProOF. Let $\mathbf{A}$ denote the interval [a, $\mathbf{b}]$ of $M^{C_{n}}$. Then obviously $\mathbf{A}$ is isomorphic to $A^{C_{n}}$. The lattice $A\left[\kappa_{A}\right]$ is $\mathbf{A} / \Phi_{A}$, where $\Phi_{A}$ is the congruence defined on $\mathbf{A}$ by the multicoloring $\kappa_{A}$. It is obvious from the definition of $\Phi$ that $\Phi_{A}$ is the restriction of $\Phi$ to $A$, from which the isomorphism follows.

\section{The main construction}

Let $D$ and $E$ be finite distributive lattices, and let

$$
\psi: D \rightarrow E
$$

be a 0 -preserving join-homomorphism. We can trivially assume that $\psi$ separates 0 (see [7]). Let $n=|\mathrm{J}(D)|, m=|\mathrm{J}(E)|, k=\max (n, m)$. We proceed in several steps.

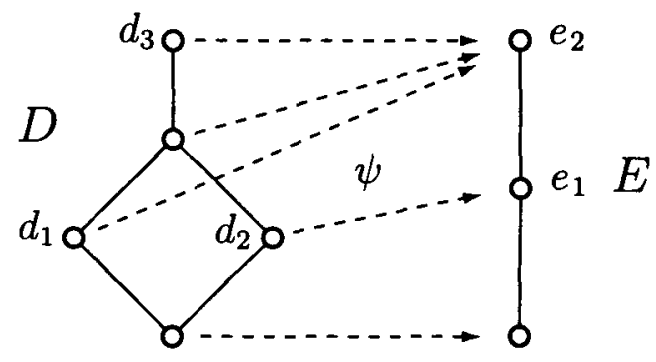

FIGURE 6. A simple example of a join-homomorphism $\psi$.

We suggest that the reader follow the construction with the example shown on Figure 6. Note that the lattice $D$ of Figure 6 is the same as the lattice $D$ of Figure 5, 
for which the small planar lattice $K$ satisfying Con $K \cong D$ is already shown in Figure 5 .

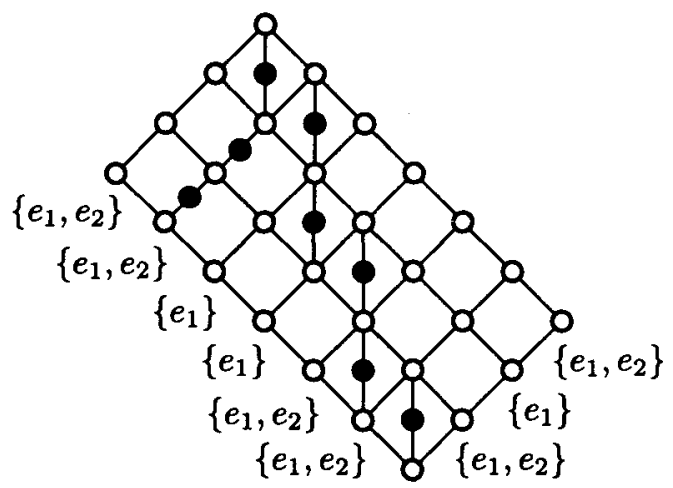

FIGURE 7. A multicolored lattice $K$.

We do the construction in four steps.

Step 1. We represent $D$ as the congruence lattice of a planar lattice $K$ as described in Section 6. To simplify the notation, we identify $D$ with Con $K$.

Step 2. We color $C_{m+1}$ with $\mathrm{J}(E)$ so that there is a bijection between the prime intervals of $C_{m+1}$ and $\mathrm{J}(E)$.

We define a map $\kappa$ of $\mathfrak{P}(K)$ to subsets of $\mathrm{J}(E)$ :

$$
\mathfrak{p} \kappa=\mathrm{J}(E) \cap(\Theta(\mathfrak{p}) \psi]_{E}=\{x \mid x \in \mathrm{J}(E), x \leq \Theta(\mathfrak{p}) \psi\}
$$

$\kappa$ is obviously isotone, $\psi$ separates 0 , so $\mathfrak{p} \kappa \neq \varnothing$. Therefore, $\kappa$ is a multicoloring of $K$ over $\mathrm{J}(E)$. (Figure 7 shows the lattice $K$ of Figure 5 multicolored with subsets of $\mathrm{J}(E)$.) Now we apply the construction in Section 7 to obtain the generalized function lattice $K[\kappa]$ with the coloring $\mu[\kappa]$.

Step 3. For every $k=\left\langle k_{0}, k_{1}\right\rangle \in K_{0}, k_{0}<1_{S_{0}}, k_{1}<1_{s_{1}}$, define the interval

$$
B_{k}=\left[\left\langle k_{0}, k_{1}\right\rangle,\left\langle k_{0}^{\dagger}, k_{1}^{\dagger}\right\rangle\right]
$$

of $K_{0}$, where $k_{0}^{\dagger}$ is the covering element of $k_{0}$ in $S_{0}$ and $k_{1}^{\dagger}$ is the covering element of $k_{1}$ in $S_{1}$. Since $K_{0}$ is a sublattice of $K$, which in turn, is a sublattice of $K[\kappa]$, it follows that $B_{k}$ defines an interval $\left(B_{k}\right)_{K}$ of $K$, and an interval $\left(B_{k}\right)_{K[k]}$ of $K[K]$. Observe that $B_{k}$ is $C_{2}^{2} ;\left(B_{k}\right)_{K}$ is $C_{2}^{2}$, or $N_{5}$, or $M_{3}$. Lemma 15 describes $\left(B_{k}\right)_{K[k]}$.

LEMMA 15. $\left(B_{k}\right)_{K[\kappa]}$ is isomorphic to $\left(\left(B_{k}\right)_{K}\right)[\kappa]$. 


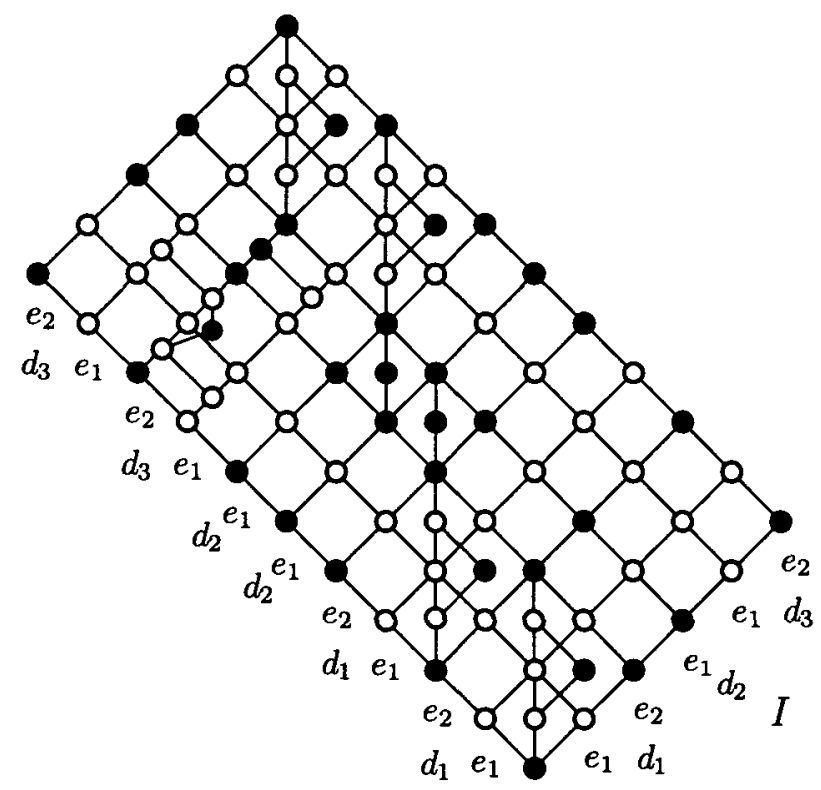

FIGURE 8. The lattice $K^{+}$.

We define a subset $K^{+}$of $K[\kappa]$ as follows, see Figure 8 (the elements of $K$ are black-filled):

$$
K^{+}=\bigcup\left(\left(B_{k}\right)_{K[\kappa]} \mid k=\left\langle k_{0}, k_{1}\right\rangle \in K_{0}, k_{0}<1_{S_{0}}, k_{1}<1_{S_{1}}\right) .
$$

Then $K^{+}$is a sublattice of $K[\kappa]$. Note that the grid $K_{0}$ is a sublattice of $K^{+}$. The extended grid $K_{0}^{\prime}$ is $K_{0}[\kappa] \cap K^{+}$, which is of the form $S_{0}^{\prime} \times S_{1}^{\prime}$, where we obtain the chain $S_{0}^{\prime}$ from $S_{0}$ by replacing a prime interval $\mathfrak{p}$ by the chain $C_{m+1, p}$ in which the prime intervals of color not in $\mathfrak{p} \kappa$ are collapsed, and similarly for $S_{1}$.

Observe that $K_{0}^{\prime} \cap\left(B_{k}\right)_{K[k]}$, the extended grid restricted to a $\left(B_{k}\right)_{K[k]}$ is a sublattice of $\left(B_{k}\right)_{K[\kappa]}$ of the form $C_{m+1} / \Phi_{0} \times C_{m+1} / \Phi_{1}$, where $\Phi_{0}$ factors out $C_{m+1}$ by the colors of $\left[k_{0}, k_{0}^{\dagger}\right] \kappa$, and $\Phi_{1}$ factors out $C_{m+1}$ by the colors of $\left[k_{1}, k_{1}^{\dagger}\right] \kappa$.

We define an ideal $I$ of $K^{+}$as the restriction of the extended grid to

$$
\left[\left\langle 0_{S_{0}}, 0_{S_{1}}\right\rangle,\left\langle 0_{S_{0}}, 1_{S_{1}}\right\rangle\right]_{K}^{+}
$$

which is a chain.

LEMMA 16. (i) $K_{0}$ is a sublattice of $K$, and $K$ is a sublattice of $K^{+}$. Moreover, $K^{+}$is a cover-preserving sublattice of $K[\kappa]$. Therefore, $K^{+}$is a colored lattice with the coloring $\mu[\kappa]$ restricted to it. 
(ii) $q \in \mathrm{J}(E)$ is the color of a prime interval of $I$ if an only if $q \leq a \psi$, for some $a \in \mathrm{J}(D)$.

(iii) For every prime interval $\mathfrak{p}$ of $K^{+}$, there is a prime interval $\mathfrak{q} \subseteq$ I of the same color (that is, $\mathfrak{p} \mu[\kappa]=\mathfrak{q} \mu[\kappa]$ ) such that $\mathfrak{p}$ and $\mathfrak{q}$ generate the same congruence in $K^{+}$.

(iv) $\left|K^{+}\right|=O\left(n^{5}\right)$.

PROOF. (i) and (ii) follow from the definitions.

(iii) Let $\mathfrak{p}$ be a prime interval of $K^{+}$; then $\mathfrak{p}$ is a prime interval of some $\left(B_{k}\right)_{K[\kappa]}$. By Lemma 7 , there is a prime interval $\mathfrak{t}$ of $B_{k}$, such that $\mathfrak{p}$ is projective to a prime interval $\mathfrak{r}$ in an edge $E_{\mathrm{t}}$ of $\left(B_{k}\right)_{K[\kappa]}$.

If $E_{\mathrm{t}}$ is a prime interval of the extended grid, then $E_{\mathrm{t}}$ is associated with a prime interval of $S_{0}^{\prime}$ or of $S_{1}^{\prime}$. In the latter case, $E_{\mathrm{t}}$ is perspective to a prime interval of $I$. In the former case, take the prime interval of $S_{0}$ that contains the prime interval of the extended grid associated with $E_{\mathrm{t}}$. By the construction of $K$, there is an $M_{3}$ in $K$ that will identify this edge with one in $I$.

If $E_{\mathfrak{t}}$ is not a prime interval of the extended grid, then $B_{k}$ is an $N_{5}$ and $t$ is $[o, a]$ or $[a, b]$ (or dually). By Lemma $7, \mathfrak{r}$ is projective to a prime interval $\mathfrak{s}$ in the maximal chain containing the interval $\left[\mathbf{o}_{n}, \mathbf{b}_{n}\right]$ of $\left(B_{k}\right)_{K[\kappa]}$. By the construction of $K$, such a prime interval projects up or down in an $N_{5}$, making it projective to a prime interval of the extended grid.

(iv) is easy, since $\left|\left(B_{k}\right)_{K[k]}\right|=O\left(m^{3}\right)$ by Lemma 8, and there are $O\left(n^{2}\right)$ such blocks by Step 1.

Step 4. We represent $E$ as the congruence lattice of a planar lattice $L_{0}$ as in Section 8 with the 'grid', $T_{0} \times T_{1}$, where $T_{1}$ is a chain of length $m=|\mathrm{J}(E)|$. We have $\left|L_{0}\right|=O\left(\mathrm{~m}^{2}\right)$. We again identify $E$ with Con $L_{0}$, and we regard $L_{0}$ as colored over $\mathrm{J}(E)$ by coloring the prime interval $\mathfrak{p}$ with $\Theta(\mathfrak{p}) \in \mathrm{J}(E)$. $L_{0}$ has a dual ideal

$$
D_{0}=\left\{\left\langle x, 1_{T_{1}}\right\rangle \mid x \in T_{0}\right\}
$$

isomorphic to $T_{0}$. We form the lattice $L_{1}=T_{0} \times I$, with the ideal

$$
I_{1}=\left\{\left\langle x, 0_{l}\right\rangle \mid x \in T_{0}\right\}
$$

isomorphic to $T_{0}$ and dual ideal

$$
D_{1}=\left\{\left\langle 1_{T_{0}}, x\right\rangle \mid x \in I\right\}
$$

isomorphic to $I$. Since both $T_{0}$ and $I$ are colored over $\mathrm{J}(E)$, there is a coloring of $L_{1}$ over $\mathrm{J}(E)$. 
We glue $L_{0}$ and $L_{1}$ together over $D_{0}$ and $I_{1}$; the resulting lattice has $D_{1}$ as a dual ideal; so we can glue this lattice together with $K^{+}$over $D_{1}$ and $I$, to obtain $L_{2}$. Since the gluing preserves the coloring, $L_{2}$ is colored over $\mathrm{J}(E)$.

Finally, we obtain the lattice $L$ from $L_{2}$ as follows: take any 'prime square' of $L_{1}$ (that is, any interval of the form $\left[\left\langle a_{0}, a_{1}\right\rangle,\left\langle b_{0}, b_{1}\right\rangle\right]$, where $\left[a_{0}, b_{0}\right]$ is a prime interval of $T_{0}$ and $\left[a_{1}, b_{1}\right]$ is a prime interval of $I$ ) that is monochromatic (that is, $\left[a_{0}, b_{0}\right]$ in $L_{0}$ and $\left[a_{1}, b_{1}\right]$ in $K^{+}$have the same color), and add an element to make the interval $\left[\left\langle a_{0}, a_{1}\right\rangle,\left\langle b_{0}, b_{1}\right\rangle\right]$ in $L$ isomorphic to $M_{3}$.

\section{Proof of Theorem 3}

Obviously, $L$ has $O\left(k^{5}\right)$ elements.

Let $\varphi$ denote the embedding of $K$ into $L$. We have to verify that $\operatorname{Con} \varphi=\psi \alpha$. It is enough to prove that $\Theta(\operatorname{Con} \varphi)=\Theta \psi \alpha$ for join-irreducible congruences $\Theta$ in $K$.

So let $\Theta=\Theta(\mathfrak{p})$, where $\mathfrak{p}=[a, b]$ is a prime interval of $K$. By Lemma 13, $\Theta(\mathfrak{p}) \operatorname{Con} \varphi=\Theta(a[\kappa], b[\kappa])$ collapses in $K[\kappa]$ the prime intervals of color $\leq \Theta \psi$; the same holds in $L_{0}$ and in $L$.

Let us assume that an element $a$ of $L$ has more than three covers. Since $L$ is glued together over chains from three lattices, $K^{+}, L_{0}$, and $L_{1}$, it follows that $a$ and its covers must be in one of these lattices. The element $a$ and its covers cannot be in $L_{0}$ because the construction in Section 8 is planar. The lattice $L_{1}$ is a direct product of two chains with some additional elements to form $M_{3}$-s, so no element of $L_{1}$ has more than three covers. Finally, if $a$ and its covers belong to $K^{+}$, then there is a largest grid element $k=\left\langle k_{0}, k_{1}\right\rangle \in K_{0}\left(k_{0}<1_{S_{0}}, k_{1}<1_{S_{1}}\right)$ contained in $a$ and then $a$ and its covers belong to $\left(B_{k}\right)_{K[\kappa]}$, which by Lemma 15 is isomorphic to $\left(\left(B_{k}\right)_{K}\right)[\kappa]$. Since $\left(B_{k}\right)_{K}$ is $C_{2}^{2}$, or $N_{5}$, or $M_{3}$, the lattice $\left(\left(B_{k}\right)_{K}\right)[\kappa]$ is of breadth 3 by Lemma 11 .

\section{Discussion}

Grätzer, Rival, and Zaguia [8] proved that the $O\left(n^{2}\right)$ result of Grätzer, Lakser, and Schmidt (see the introduction) is 'best possible' in the sense that in Theorem 1 size $O\left(n^{2}\right)$ cannot be replaced by size $O\left(n^{\alpha}\right)$, for any $\alpha<2$. This was improved in Zhang [14] and in Grätzer and Wang [10].

There are two crucial questions left open in this paper.

The first question is whether $O\left(k^{5}\right)$ is the optimal size for the lattice $L$ in Theorem3. Can one prove (analogously to Grätzer, Rival, and Zaguia [8]) that size $O\left(k^{5}\right)$ cannot be replaced by size $O\left(k^{\alpha}\right)$, for any $\alpha<5$ ? Can one find a lower bound for $|L|$ as in the result of Grätzer and Wang [10]? 
The second question is whether breadth 3 is optimal for $L$ ? This is almost certainly so since a breadth 2 lattice cannot contain a $C_{2}^{3}$, making it very difficult to direct the congruences.

It seems to us that the lattice $L$ we construct in this paper is of order dimension three. It would be interesting to prove this.

Although this whole paper deals with the construction of the lattice $L$, it should be pointed out that we could not have started with a different $K$. The properties of the lattice $K$ (borrowed from Grätzer, Lakser, and Schmidt [5]) are crucial for the construction of $L$. Can one construct $L$ starting from a different lattice $K$ ?

\section{References}

[1] D. Duffus, B. Jónsson and I. Rival, 'Structure results for function lattices', Canad. J. Math. 33 (1978), 392-400.

[2] N. Funayama and T. Nakayama, 'On the distributivity of a lattice of lattice-congruences', Proc. Imp. Acad. Tokyo 18 (1942), 553-554.

[3] G. Grätzer, General lattice theory, 2nd ed. (Birkhäuser Basel, 1998).

[4] G. Grätzer and H. Lakser, 'Congruence lattices of planar lattices', Acta Math. Hungar. 60 (1992), 251-268.

[5] G. Grätzer, H. Lakser and E. T. Schmidt, 'Congruence lattices of small planar lattices', Proc. Amer. Math. Soc. 123 (1995), 2619-2623.

[6] — - 'Congruence representations of .join homomorphisms of distributive lattices: A short proof', Math. Slovaca 46 (1996), 363-369.

[7] - 'Isotone maps as maps of congruences. I. Abstract maps', Acta Math. Acad. Sci. Hungar. 75 (1997), 81-111.

[8] G. Grätzer, I. Rival and N. Zaguia, 'Small representations of finite distributive lattices as congruence lattices', Proc. Amer. Math. Soc. 123 (1995), 1959-1961.

[9] G. Grätzer and E. T. Schmidt, 'On congruence lattices of lattices', Acta Math. Acad. Sci. Hungar. 13 (1962), 179-185.

[10] G. Grätzer and D. Wang, 'A lower bound for congruence representations', Order 14 (1997), 67-74.

[11] A. P. Huhn, 'On the representation of distributive algebraic lattices. I-III', Acta Sci. Math. (Szeged) 45 (1983), 239-246; 53 (1989), 3-10, 11-18.

[12] E. T. Schmidt, 'Zur Charakterisierung der Kongruenzverbände der Verbände', Mat. Časopis Sloven. Akad. Vied. 18 (1968), 3-20.

[13] S.-K. Teo, 'On the length of the congruence lattice of a lattice', Period. Math. Hungar. 21 (1990), 179-186.

[14] Y. Zhang, 'A note on 'Small representations of finite distributive lattices as congruence lattices", Order 13 (1996), 365-367.

Department of Mathematics

University of Manitoba

Winnipeg, Man. R3T 2N2

Canada
Mathematical Institute Technical University of Budapest Múegyetem rkp. 3 H-1521 Budapest Hungary 